\title{
Conformal Switched Beam Antenna
}

\author{
S. Swaisaenyakorn, A.J. Cole, C.C.H. Ng, P.R. Young \\ School of Engineering and Digital Arts \\ University of Kent \\ Canterbury, United Kingdom \\ ajc87@kent.ac.uk
}

\begin{abstract}
A cylindrical switched beam antenna making use of Kapton film and aperture-coupling is presented. The use of four elements controlled via a high frequency switching stage is proposed. Using very simple bias circuitry, switching can be achieved to provide four-way coverage at $0^{\circ}, 90^{\circ}, 180^{\circ}$ and $270^{\circ}$. Elements consist of an aperture-coupled patch making use of thin film technology to provide an air substrate for increased efficiency and therefore gain. Four direction beam switching is performed using only three switches integrated into the structure. Simulated results of a single element are presented, followed by measured results of the fully fabricated structure.
\end{abstract}

Keywords-switched-beam; patch; kapton; thin-film; aperturecoupled; antenna; air substrate.

\section{INTRODUCTION}

The modern wireless environment requires ever increasing coverage. One of the main challenges in meeting this requirement is the availability of spectrum, where bandwidth is directly proportional to maximum channel capacity. Switched beam antennas make use of spatial diversity, which can vastly reduce issues associated with multipath environments while increasing spectral efficiency through frequency reuse [1, 2]. For further wireless communications standards where higher frequency spectrum is utilized, we find that adaptive, directional antennas are a necessity [3].

Beam steering has been classically achieved through the use of phased arrays, where individual antenna elements are driven with phase differences. However, the implementation of such designs can become complex, especially for larger arrays, and requires a significant amount of space. For a much simpler approach, it is possible to produce a switching network to switch between directional antennas. This method has the distinct advantage of reduced complexity as well as increased control over the individual beams. By using a number of antennas, we also know the radiation pattern should be highly similar in each direction, and only the switching network itself should have an impact.

The implementation of a switching network has been used in [4], where the authors utilized a monopole slot antenna. Similarly in [5], where instead a planar quasi-Yagi-Uda array is used as the antenna element.

It is possible to further reduce the size of a switched beam device by incorporating the beam switching into a single antenna structure. This was performed in [6] through the use of pin diodes which switched the radiating apertures of a patch antenna on or off. Additional work was carried out in [7], where the authors designed a single switchable structure with a six direction switched beam. The device was characterized by a substrate integrated waveguide (SIW) featuring four PIN diode loaded slots and a reflector element.

\section{PRINCIPLE OF OPERATION}

In this paper we present the idea of using three ultrawideband (UWB) switches to route power to four antennas positioned evenly around the circumference of a standard polyvinyl chloride (PVC) pipe. Each element can be activated through simple bias circuitry, which could be controlled by equally simple logic with ample space for full integration into the structure.

The design makes use of thin film technology to enable the suspension of each antenna element over an air gap, allowing for a low loss, low permittivity substrate. The patch antennas are fed via aperture coupling, permitting the switching network to be isolated from the radiating elements.

\section{APERTURE COUPLED PATCH ANTENNAS}

The proposed design uses four aperture coupled patch elements, making use of Kapton film to achieve an air substrate increasing the potential for high gain. A PVC pipe cut with four holes provides the support structure, with an antenna positioned over each hole.

Aperture coupling has been chosen as the patch feeding technique due the option for two substrates, one each for the feeding network and radiating side. Substrate types and dimensions can be chosen appropriately to optimize performance. Using a thin dielectric between the ground plane and microstrip feed reduces spurious radiation, and a thicker, lower permittivity substrate on the radiating side improves gain by increasing efficiency. The geometry of the coupling technique can be seen in Fig. 1 and Fig. 2, where air and Kapton have been chosen as the radiating and feed substrates, respectively. The air substrate is achieved by etching the patch on Kapton, and then placing this film over a rectangular cut in a PVC pipe. The ground aperture width can be chosen such that sufficient energy is coupled to the patch, while minimizing the amount of backscattered radiation, and its length can perform impedance matching [8]. The stub length, $S t u b_{L}$, is able to tune out the reactance introduced by the ground aperture. This can be optimized during simulation. 


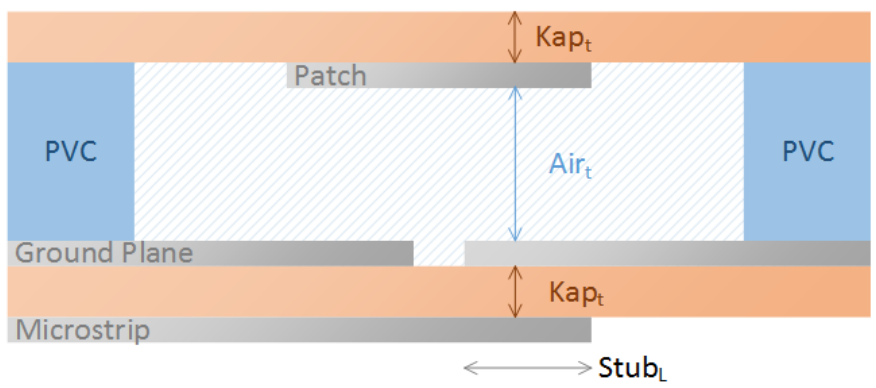

Fig. 1. Cross-sectional view of an aperture coupled patch

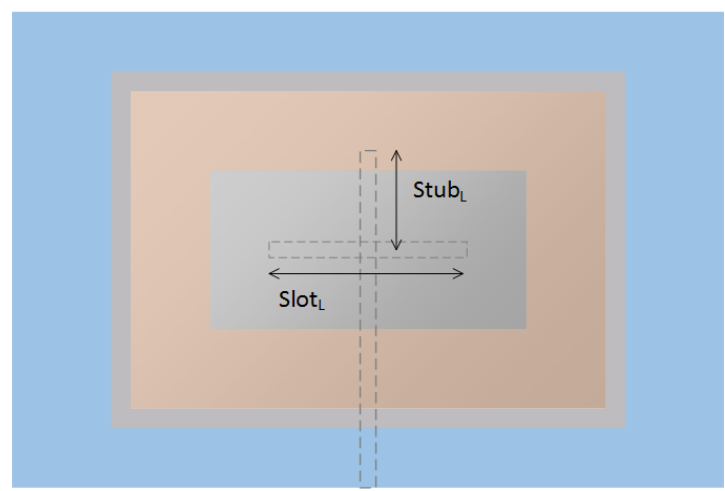

Fig. 2. Plan view of radiating element

\section{DESIGN PROCESS \& IMPLEMENTATION}

The structure has been designed to operate at $6 \mathrm{GHz}$, where each patch has dimensions of $18.5 \times 37 \mathrm{~mm}$, with a corresponding ground aperture of $2 \times 18 \mathrm{~mm}$. The microstrip feed line has a width of $290 \mu \mathrm{m}$ on $125 \mu \mathrm{m}$ Kapton film with $\varepsilon_{\mathrm{r}}=3.4, \tan \delta=0.002$ and a copper thickness of $18 \mu \mathrm{m}$. The feed width corresponds with $50 \Omega$, and allows the entire switching network to have the same characteristic impedance, ensuring good matching with the three switches.

One aperture coupled patch was simulated in CST, using a $50 \Omega$ port as a switch output. A planar antenna was simulated, then curved to the appropriate $50 \mathrm{~mm}$ radius of the PVC pipe, see Fig. 3 (the patch is shown on top of the Kapton for clarity). The simulated S11 magnitude can be seen in Fig. 4, where there is little difference, however, we can assume the slight change is due to a difference in discretization between the planar and curved structure within CST. A comparison of the normalized radiation patterns at $6 \mathrm{GHz}$ for each antenna can be seen in Fig. 5, where the dotted line denotes the planar device. The planar and curved antennas achieve a peak gain of $10.1 \mathrm{dBi}$ and $9.55 \mathrm{dBi}$, respectively. Simulations differed negligibly when the other antennas were introduced, as expected. Because aperture coupled patches are well understood, it is assumed that the difficulties with this device are related to its fabrication.

Fig. 6 displays the practical implementation of one of the antenna elements, along with a birds eye view of the switched beam directions; the microstrip feed width is exaggerated for clarity. First, a window is cut from the PVC pipe, allowing adequate clearance to reduce perturbation of the radiating fields. A Kapton film with the etched patch is applied to the outside of the pipe using adhesive tape, attempting to ensure the antenna remains conformal with the pipe circumference. This process is repeated three times for the other antennas. The inside of the pipe is applied with a single piece of Kapton etched with the ground plane and apertures on one side, along with the microstrip feed lines and switch circuitry on the other.

We have chosen the Skyworks SKY13298-360LF UWB three port switch to implement the switching between each antenna, requiring only three devices. The initial switching stage at the signal input routes power to one of two antenna pairs, where a further switch for each pair routes the signal to an individual antenna, where only one element is active at any time. Fig. 7 shows a portion of the switching network on the inside of the pipe. The device can be biased at $3.3 \mathrm{~V}$, and a switch box was constructed for beam switching in measurements. The final structure can be seen in Fig. 8, at 100mm in diameter.
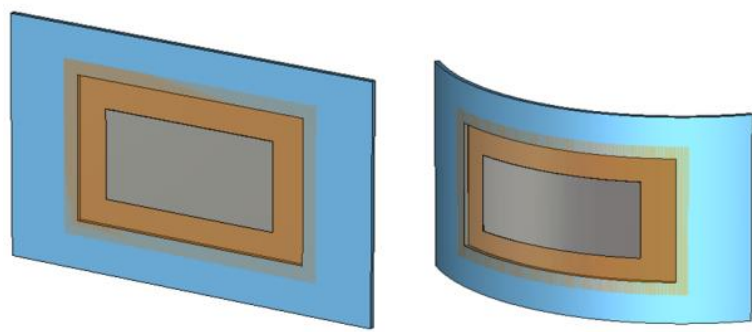

Fig. 3. Planar and curved aperture coupled patch

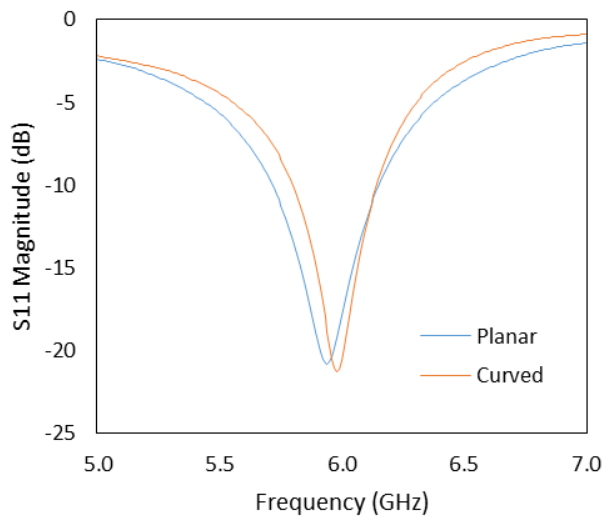

Fig. 4. Simulated S11 magnitude for planar and curved antenna

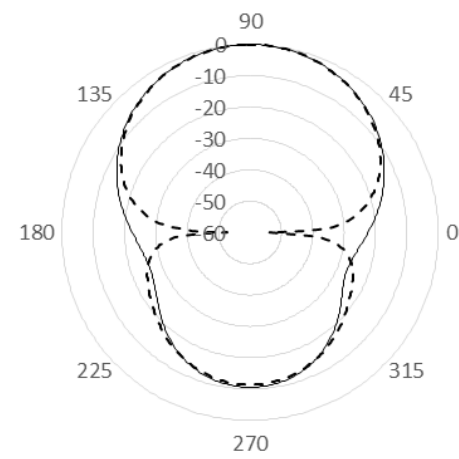

Fig. 5. Normalized radiation patterns for planar (dotted) and curved antennas 

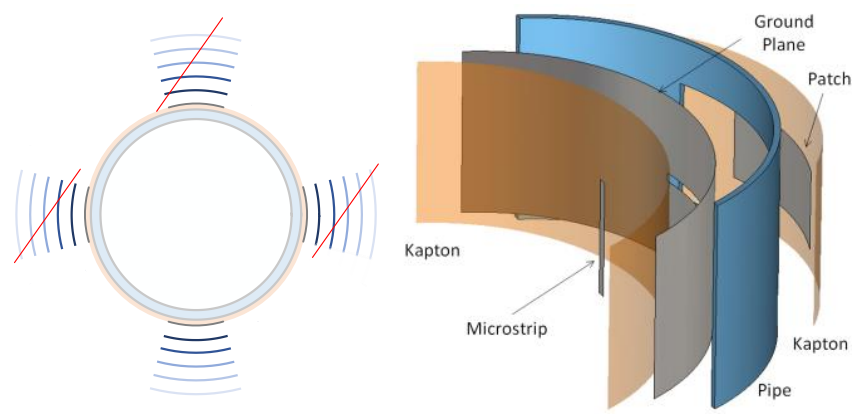

Fig. 6. Birds eye view and exploded view of a curved, single antenna element

\section{Discussion of RESUlTS}

Fig. 9 shows the measured result for each switched beam. The peak antenna resonances show some disagreement with simulated results. It can be seen that each element operates at around $6.4-6.45 \mathrm{GHz}$, with $\mathrm{S} 11$ magnitudes between -31 and $-37 \mathrm{~dB}$. The structure does appear to be lossy, showing reflections below $-10 \mathrm{~dB}$ across some of the frequency range. It should be noted that the switches have an impact due to their respective insertion and return loss characteristics [9]. Also, the switching network is comprised of relatively long microstrip lines, which could well contribute to the measured response. A further simulation was performed to include a 12 $\mathrm{cm}$ length of microstrip, as well as a decreased air gap to account for the slight depression of the Kapton film into the cavity. The simulated result shown in Fig. 9 shows a much better match with the measurements.

Following measurement of the S-parameters, a custom jig was constructed to mount the antenna structure in an anechoic chamber for radiation pattern measurements. The measurements were taken at $6.45 \mathrm{GHz}$ and the normalized patterns can be seen for each switched beam in Fig. 10 .

The radiation patterns show good results with definitive four direction coverage. The individual directionality of each antenna is somewhat arbitrary due to the mounting constraints within the chamber, but the relative switched beam direction between each element shows a $90^{\circ}$ difference. An attempt has not been made to compare simulated radiation patterns to measured results for two reasons. The switches would be difficult to model accurately because of their unknown internal parameters, and additionally, due to the symmetry of simulation, the comparison between simulated switched beams would show identical patterns in each direction.

\section{CONCLUSION}

This paper has demonstrated a switched beam antenna with almost $360^{\circ}$ coverage over four discrete directions. It has shown how this can be achieved with only three switches, ensuring a relatively low-cost design. The measured results show good agreement with simulations at the operating frequency despite the effect of switch characteristics. Due to the conformal nature of the switched beam antenna, it could be adapted to a variety of shapes, and would easily adapt to cylinders of differing radii. The structure could have potential at higher frequencies as smaller antenna elements would allow more room for the extension of coverage in $n$ directions, depending on the antenna and support structure size; an increased number of switches would be required. Smaller antenna elements could also have the capability of higher gain operation by introducing, for example, $2 \times 2$ square arrays with an alternative feeding method, in place of a single, lower frequency counterpart. Alternatively, the four direction switched beam antenna could simply see a reduction in the size of the structure at higher frequencies

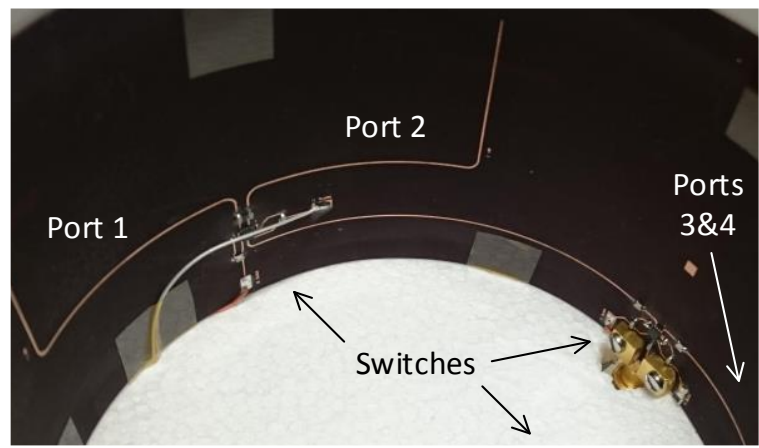

Fig. 7. Internal view of the switching network

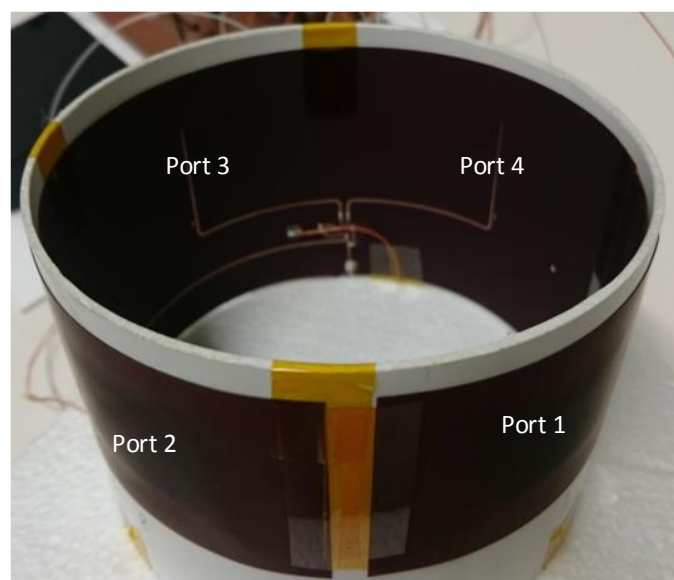

Fig. 8. Completed switched beam structure

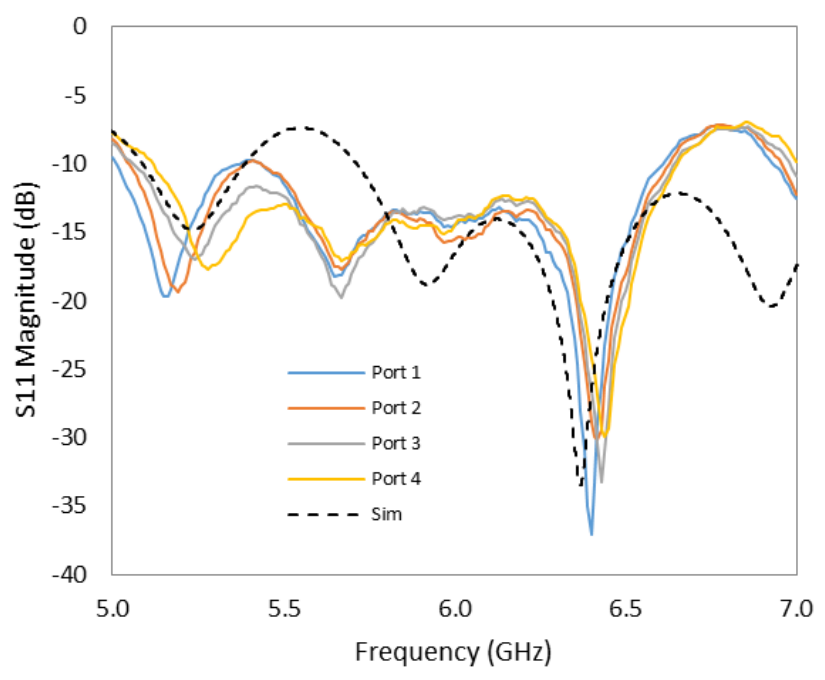

Fig. 9. Measured S11 for all ports 


\section{REFERENCES}
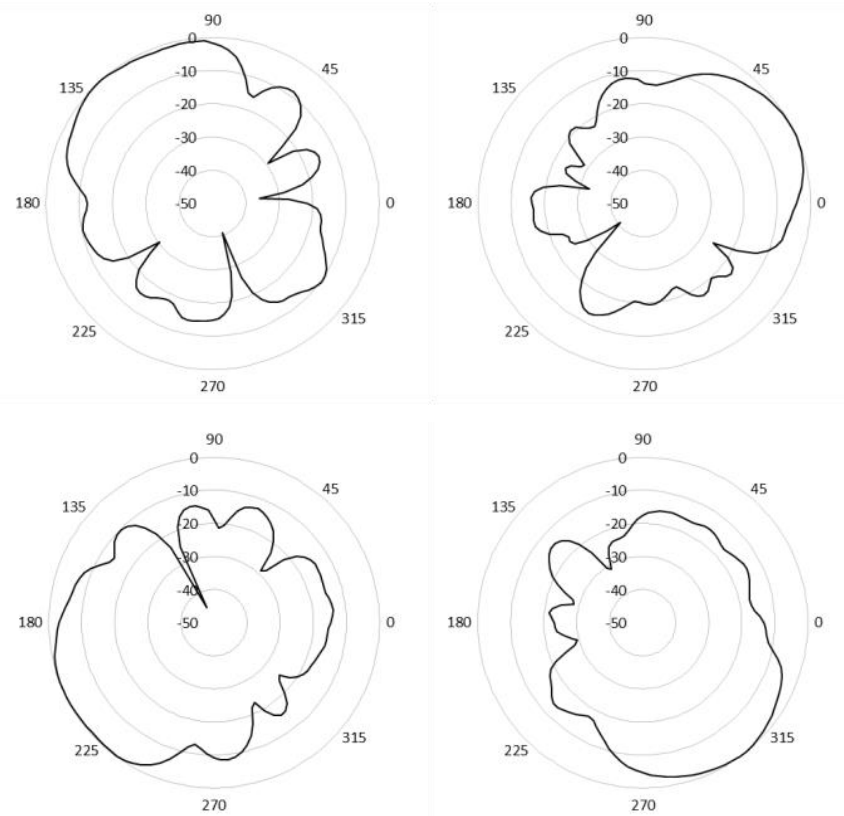

Fig. 10. Four direction normalized measured radiation pattern. Antenna element clockwise from top left: $3,4,1,2$.
[1] M. Chryssomallis, "Smart antennas," IEEE Magazine Antennas Propag., vol. 42, pp. 129 - 136, 2000.

[2] J. H. Winters, "Smart antennas for wireless systems," IEEE Pers. Commun., vol. 5, pp. 23 - 27, 1998.

[3] K. Huang and D. J. Edwards, "Millimetre Wave Antennas for Gigabit Wireless Communications: A Practical Guide to Design and Analysis in a System Context". Wiley-Blackwell, 2008.

[4] Ming-Iu Lai, Tzung-Yu Wu, Jung-Chin Hsieh, Chun-Hsiung Wang and Shyh-Kang Jeng, "Compact Switched-Beam Antenna Employing a Four- Element Slot Antenna Array for Digital Home Applications," IEEE Trans. Antennas Propag., vol. 56, no.9, pp. 2929 - 2936, 2008.

[5] R. A. Alhalabi, Yi-Chyun Chiou and G. M. Rebeiz, "Self-Shielded High- Efficiency Yagi-Uda Antennas for $60 \mathrm{GHz}$ Communications," IEEE Trans. Antennas Propag., vol. 59, no.3 , pp. 742 - 750, 2011.

[6] P. Ngamjanyaporn and M. Krairiksh, "Switched-beam single patch antenna," IEEE Electron. Lett., vol. 38, no.1 , pp. 7 - 8, 2002.

[7] L. Wu, A. J. Farrall and P. R. Young, "Substrate Integrated Waveguide Switched Beam Antenna," IEEE Trans. Antennas Propag., vol. 63, no. 5, pp. $2301-2305,2015$.

[8] D. Pozar, "Microstrip Antennas," IEEE Proc., vol. 80, no. 1, pp. $79-$ 91, 1992.

[9] Skyworks, "GaAs SP2T Switch for Ultra Wideband (UWB) 3-8 GHz," SKY13298-360LF datasheet, Jul. 2008. 